\title{
EIGENFREQUENCIES OF GENERALLY RESTRAINED BEAMS
}

\author{
RICARDO OSCAR GROSSI \\ AND CARLOS MARCELO ALBARRACÍN
}

Received 13 March 2002 and in revised form 15 May 2003

We deal with the exact determination of eigenfrequencies of a beam with intermediate elastic constraints and generally restrained ends. It is the purpose of this paper to use the calculus of variations to obtain the equations of motion and the natural boundary conditions, and particularly those at the intermediate constraints. Numerical values for the first five natural frequencies are presented in a tabular form for a wide range of values of the restraint parameters. Several particular cases are presented and some of these cases have been compared with those available in the literature.

\section{Introduction}

Several investigators have studied the influence of rotational and/or translational restraints at the ends of vibrating beams $[2,3,4,5,6,8$, $9,10,11,12,14,15,16,17,18,19,20]$. Rao and Mirza [22] have derived exact frequency and normal mode shape expressions for uniform beams with ends elastically restrained against rotation and translation. Nallim and Grossi [21] studied the dynamical behaviour of beams with complicating effects such as nonuniform cross sections, presence of an arbitrarily placed concentrated mass and an axial force, and ends elastically restrained against rotation and translation.

In contrast to the body of information described, there is only a limited amount of information for beams elastically restrained at intermediate points. Rutemberg [24] presented eigenfrequencies for a uniform cantilever beam with a rotational restraint at some position. Lau [13] extended Rutemberg's results including an additional spring to against 
504 Eigenfrequencies of generally restrained beams

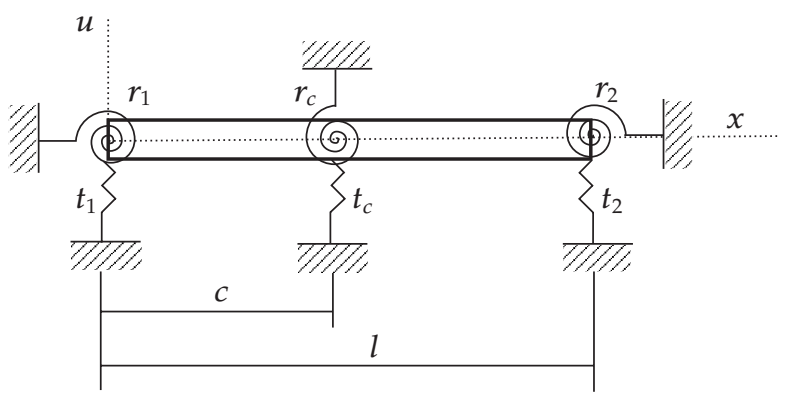

FIGURE 2.1. Vibrating system under study.

translation. Arenas and Grossi [1] presented exact and approximate frequencies of a uniform beam, with one end spring-hinged and a rotational restraint in a variable position.

The present paper is concerned with the general problem of free vibrations of a uniform beam with intermediate constraints and ends elastically restrained against rotation and translation.

Exact expressions for frequencies are presented. The generally restrained beam analysed includes the classical end conditions: clamped, simply supported, sliding, and free as simply particular cases. It also includes the cases with ends and/or intermediate points elastically restrained, previously analysed by other investigators and available in the literature. Some of these particular cases are discussed.

The eigenvalues have been calculated numerically by applying a bracketing method strategy to the corresponding frequency equation. Results for the first five eigenfrequencies for some typical cases are presented. A comparison with published results is included. A great number of problems were solved; and since this number of cases is prohibitively large, results are presented for only a few cases.

\section{Variational derivation of the boundary and eigenvalue problem}

We consider the uniform beam of length $l$, shown in Figure 2.1, which has elastically restrained ends and is constrained at an intermediate point with variable position. It has been assumed that the ends and the intermediate points are elastically restrained against rotation and translation. The rotational restraints are characterised by the spring constants $r_{1}, r_{2}$, and $r_{c}$ and the translational restraints by the spring constants $t_{1}, t_{2}$, and $t_{c}$. Adopting the adequate values of the parameters $r_{i}$ and $t_{i}, i=1,2$, all the possible combinations of classical end conditions (i.e., clamped, pinned, sliding, and free) can be generated. On the other hand, adopting 
the adequate values of the parameters $r_{c}$ and $t_{c}$, different constraints can be generated and also the case of a two-span beam.

It is the purpose of this paper to use the calculus of variations to obtain the equations of motion and the natural boundary conditions, particularly, those at the intermediate constraints.

In order to analyse the transverse planar displacements of the system under study, we suppose that the vertical position of the beam at any time $t$ is described by the function $u=u(x, t), x \in[0, l]$.

It is well known that at time $t$, the kinetic energy of the beam, the potential energy due to elastic deformation of the beam, and the springs are given by (see $[7,25])$

$$
\begin{aligned}
T= & \frac{1}{2} \int_{0}^{l} \rho A\left(\frac{\partial u(x, t)}{\partial t}\right)^{2} d x \\
U= & \frac{1}{2}\left\{\int_{0}^{l} \mathrm{EI}\left(\frac{\partial^{2} u(x, t)}{\partial x^{2}}\right)^{2} d x+r_{1}\left(\frac{\partial u(0, t)}{\partial x}\right)^{2}+t_{1} u(0, t)^{2}\right. \\
& \left.\quad+r_{c}\left(\frac{\partial u(c, t)}{\partial x}\right)^{2}+t_{c} u(c, t)^{2}+r_{2}\left(\frac{\partial u(l, t)}{\partial x}\right)^{2}+t_{2} u(l, t)^{2}\right\},
\end{aligned}
$$

where $\rho$ is the mass per unit length, $A$ the cross-sectional area, and EI the flexural rigidity of the beam.

Hamilton's principle requires that between times $t_{a}$ and $t_{b}$, at which the positions are known, the motion will make the action integral $F(u)=$ $\int_{t_{a}}^{t_{b}} L d t$ on the space of admissible functions stationary, where the Lagrangian is given by $L=T-U$ (see [26]).

In consequence, the energy functional to be considered is given by

$$
\begin{aligned}
F(u)= & \frac{1}{2} \int_{t_{a}}^{t_{b}} \int_{0}^{l}\left(\rho A\left(\frac{\partial u(x, t)}{\partial t}\right)^{2}-\mathrm{EI}\left(\frac{\partial^{2} u(x, t)}{\partial x^{2}}\right)^{2}\right) d x d t \\
& -\frac{1}{2} \int_{t_{a}}^{t_{b}} r_{1}\left(\frac{\partial u(0, t)}{\partial x}\right)^{2} d t-\frac{1}{2} \int_{t_{a}}^{t_{b}} t_{1} u(0, t)^{2} d t-\frac{1}{2} \int_{t_{a}}^{t_{b}} r_{c}\left(\frac{\partial u(c, t)}{\partial x}\right)^{2} d t \\
& -\frac{1}{2} \int_{t_{a}}^{t_{b}} t_{c} u(c, t)^{2} d t-\frac{1}{2} \int_{t_{a}}^{t_{b}} r_{2}\left(\frac{\partial u(l, t)}{\partial x}\right)^{2} d t-\frac{1}{2} \int_{t_{a}}^{t_{b}} t_{2} u(l, t)^{2} d t
\end{aligned}
$$

The stationary condition for the functional (2.2) requires that $\delta F(u, v)=$ 0 , for all $v \in \mathbf{D}_{0}$, where $\mathbf{D}_{0}$ is the space of admissible directions at $u$ for the domain $\mathbf{D}$ of the functional. 
506 Eigenfrequencies of generally restrained beams

In order to make the mathematical developments required by the application of the techniques of the calculus of variations, we assume that the domain $\mathbf{D}$ is the set of functions

$$
u(x, \cdot) \in C^{2}\left[t_{a}, t_{b}\right], \quad u(\cdot, t) \in C^{1}[0, l] \cap \hat{C}^{4}[0, l]
$$

where $\hat{C}^{4}[0, l]$ denotes the space of functions with piecewise continuous derivatives up to order four with only one corner point $c$. At this point $c$, at least the one-sided derivatives, with respect to $x$ of order greater than one, exist. For instance, $\partial^{2} u(x, t) / \partial x^{2}$ is continuous on $[0, l]$ except at the point $c$, where it has the one-sided derivatives $\partial^{2} u\left(c^{-}, t\right) / \partial x^{2}$ and $\partial^{2} u\left(c^{+}, t\right) / \partial x^{2}$. The same situation occurs for $\partial^{3} u(x, t) / \partial x^{3}$ and $\partial^{4} u(x, t) /$ $\partial x^{4}$. Consequently, if $u(\cdot, t) \in C^{1}[0, l] \cap \hat{C}^{4}[0, l]$, then $u(\cdot, t) \in C^{1}[0, l]$ and also $u \in C^{4}[0, c]$ and $u \in C^{4}[c, l]$.

In view of all these observations and since Hamilton's principle requires that between times $t_{a}$ and $t_{b}$, the positions are known, the domain of the functional (2.2) is given by

$$
\begin{aligned}
& \mathbf{D}=\left\{u: u(x, \cdot) \in C^{2}\left[t_{a}, t_{b}\right], u(\cdot, t) \in C^{1}[0, l] \cap \hat{C}^{4}[0, l],\right. \\
&\left.u\left(x, t_{a}\right), u\left(x, t_{b}\right) \text { prescribed }\right\} .
\end{aligned}
$$

Since $u(\cdot, t) \in C^{1}[0, l]$, there exists continuity of deflection and slope at the point $x=c$ and this generates the following conditions:

$$
\begin{gathered}
u\left(c^{-}, t\right)=u\left(c^{+}, t\right)=u(c, t), \\
\frac{\partial u\left(c^{-}, t\right)}{\partial x}=\frac{\partial u\left(c^{+}, t\right)}{\partial x}=\frac{\partial u(c, t)}{\partial x} .
\end{gathered}
$$

The only admissible directions $v$ at $u \in \mathbf{D}$ are those for which $u+\varepsilon v \in \mathbf{D}$ for sufficiently small $\varepsilon$; in consequence, in view of (2.4), $v$ is an admissible direction at $u$ for $\mathbf{D}$ if and only if $v \in \mathbf{D}_{0}$, where

$$
\begin{gathered}
\mathbf{D}_{0}=\left\{v: v(x, \cdot) \in C^{2}\left[t_{a}, t_{b}\right], v(\cdot, t) \in C^{1}[0, l] \cap \hat{C}^{4}[0, l],\right. \\
\left.v\left(x, t_{a}\right)=v\left(x, t_{b}\right)=0, \forall x \in(0, l)\right\} .
\end{gathered}
$$

Using the definition of variation of $F$ at $u$ in the direction $v$

$$
\delta F(u ; v)=\left.\frac{d F(u+\varepsilon v)}{d \varepsilon}\right|_{\varepsilon=0},
$$


we obtain

$$
\begin{aligned}
\delta F(u ; v)= & \int_{t_{a}}^{t_{b}} \int_{0}^{l}\left(\rho A \frac{\partial u}{\partial t} \frac{\partial v}{\partial t}-\mathrm{EI} \frac{\partial^{2} u}{\partial x^{2}} \frac{\partial^{2} v}{\partial x^{2}}\right) d x d t \\
& -\int_{t_{a}}^{t_{b}} r_{1} \frac{\partial u(0, t)}{\partial x} \frac{\partial v(0, t)}{\partial x} d t-\int_{t_{a}}^{t_{b}} t_{1} u(0, t) v(0, t) d t \\
& -\int_{t_{a}}^{t_{b}} r_{c} \frac{\partial u(c, t)}{\partial x} \frac{\partial v(c, t)}{\partial x} d t-\int_{t_{a}}^{t_{b}} t_{c} u(c, t) v(c, t) d t \\
& -\int_{t_{a}}^{t_{b}} r_{2} \frac{\partial u(l, t)}{\partial x} \frac{\partial v(l, t)}{\partial x} d t-\int_{t_{a}}^{t_{b}} t_{2} u(l, t) v(l, t) d t .
\end{aligned}
$$

Now we consider the integral $\int_{t_{a}}^{t_{b}} \int_{0}^{l}(\rho A(\partial u / \partial t)(\partial v / \partial t)) d x d t$. Since $u(x, \cdot)$, $v(x, \cdot) \in C^{2}\left[t_{a}, t_{b}\right]$, we can integrate by parts with respect to $t$; and if we apply the conditions $v\left(x, t_{a}\right)=v\left(x, t_{b}\right)=0$ for all $x \in(0, l)$, imposed in (2.6), we obtain

$$
\int_{t_{a}}^{t_{b}} \int_{0}^{l} \rho A \frac{\partial u}{\partial t} \frac{\partial v}{\partial t} d x d t=-\int_{t_{a}}^{t_{b}} \int_{0}^{l} \rho A \frac{\partial^{2} u}{\partial t^{2}} v d x d t
$$

On the other hand, in $\int_{t_{a}}^{t_{b}} \int_{0}^{l} \operatorname{EI}\left(\partial^{2} u / \partial x^{2}\right)\left(\partial^{2} v / \partial x^{2}\right) d x d t$, the integrand may not be continuous at the corner point $c$, but since

$$
u(\cdot, t), v(\cdot, t) \in C^{4}[0, c], \quad u(\cdot, t), v(\cdot, t) \in C^{4}[c, l]
$$

the integral may be represented as the sum of two integrals on $[0, c]$ and $[c, l]$, respectively. Thus if we integrate twice by parts, with respect to $x$, we obtain

$$
\begin{aligned}
& \int_{t_{a}}^{t_{b}} \int_{0}^{c} \mathrm{EI} \frac{\partial^{2} u}{\partial x^{2}} \frac{\partial^{2} v}{\partial x^{2}} d x d t \\
&=\int_{t_{a}}^{t_{b}} \int_{0}^{c} \mathrm{EI} \frac{\partial^{4} u}{\partial x^{4}} v d x d t \\
& \quad+\mathrm{EI} \int_{t_{a}}^{t_{b}}\left(\frac{\partial^{2} u\left(c^{-}, t\right)}{\partial x^{2}} \frac{\partial v(c, t)}{\partial x}-\frac{\partial^{2} u(0, t)}{\partial x^{2}} \frac{\partial v(0, t)}{\partial x}\right. \\
&\left.\quad-\frac{\partial^{3} u\left(c^{-}, t\right)}{\partial x^{3}} v(c, t)+\frac{\partial^{3} u(0, t)}{\partial x^{3}} v(0, t)\right) d t
\end{aligned}
$$


508 Eigenfrequencies of generally restrained beams

Similarly, we obtain

$$
\begin{aligned}
& \int_{t_{a}}^{t_{b}} \int_{c}^{l} \mathrm{EI} \frac{\partial^{2} u}{\partial x^{2}} \frac{\partial^{2} v}{\partial x^{2}} d x d t \\
& =\int_{t_{a}}^{t_{b}} \int_{c}^{l} \mathrm{EI} \frac{\partial^{4} u}{\partial x^{4}} v d x d t \\
& \quad+\mathrm{EI} \int_{t_{a}}^{t_{b}}\left(-\frac{\partial^{2} u\left(c^{+}, t\right)}{\partial x^{2}} \frac{\partial v(c, t)}{\partial x}+\frac{\partial^{2} u(l, t)}{\partial x^{2}} \frac{\partial v(l, t)}{\partial x}\right. \\
& \left.\quad+\frac{\partial^{3} u\left(c^{+}, t\right)}{\partial x^{3}} v(c, t)-\frac{\partial^{3} u(l, t)}{\partial x^{3}} v(l, t)\right) d t .
\end{aligned}
$$

Replacing (2.9), (2.11), and (2.12) in (2.8), we obtain

$$
\begin{aligned}
\delta F(u ; v)= & -\int_{t_{a}}^{t_{b}} \int_{0}^{c}\left(\rho A \frac{\partial^{2} u}{\partial t^{2}}+\mathrm{EI} \frac{\partial^{4} u}{\partial x^{4}}\right) v d x d t \\
& -\int_{t_{a}}^{t_{b}} \int_{c}^{l}\left(\rho A \frac{\partial^{2} u}{\partial t^{2}}+\mathrm{EI} \frac{\partial^{4} u}{\partial x^{4}}\right) v d x d t \\
& +\int_{t_{a}}^{t_{b}}\left(-r_{1} \frac{\partial u(0, t)}{\partial x}+\mathrm{EI} \frac{\partial^{2} u(0, t)}{\partial x^{2}}\right) \frac{\partial v(0, t)}{\partial x} d t \\
& +\int_{t_{a}}^{t_{b}}\left(-t_{1} u(0, t)-\mathrm{EI} \frac{\partial^{3} u(0, t)}{\partial x^{3}}\right) v(0, t) d t \\
& +\int_{t_{a}}^{t_{b}}\left(-r_{c} \frac{\partial u(c, t)}{\partial x}-\mathrm{EI} \frac{\partial^{2} u\left(c^{-}, t\right)}{\partial x^{2}}+\mathrm{EI} \frac{\partial^{2} u\left(c^{+}, t\right)}{\partial x^{2}}\right) \frac{\partial v(c, t)}{\partial x} d t \\
& +\int_{t_{a}}^{t_{b}}\left(-t_{c} u(c, t)+\mathrm{EI} \frac{\partial^{3} u\left(c^{-}, t\right)}{\partial x^{3}}-\mathrm{EI} \frac{\partial^{3} u\left(c^{+}, t\right)}{\partial x^{3}}\right) v(c, t) d t \\
& +\int_{t_{a}}^{t_{b}}\left(-r_{2} \frac{\partial u(l, t)}{\partial x}-\mathrm{EI} \frac{\partial^{2} u(l, t)}{\partial x^{2}}\right) \frac{\partial v(l, t)}{\partial x} d t \\
& +\int_{t_{a}}^{t_{b}}\left(-t_{2} u(l, t)+\mathrm{EI} \frac{\partial^{3} u(l, t)}{\left.\partial x^{3}\right) v(l, t) d t .}\right.
\end{aligned}
$$

According to Hamilton's principle, the expression (2.13) must vanish for the function $u$ corresponding to the actual motion of the beam. If we first suppose that both ends of the beam and the restraint at $x=c$ are rigidly clamped, the directions $v$ must satisfy

$$
v(0, t)=\frac{\partial v(0, t)}{\partial x}=v(l, t)=\frac{\partial v(l, t)}{\partial x}=v(c, t)=\frac{\partial v(c, t)}{\partial x}=0 \quad \forall t \in\left(t_{a}, t_{b}\right) .
$$


Using (2.14) in (2.13) leads to

$$
\begin{aligned}
\delta F(u ; v)= & -\int_{t_{a}}^{t_{b}} \int_{0}^{c}\left(\rho A \frac{\partial^{2} u}{\partial t^{2}}+\mathrm{EI} \frac{\partial^{4} u}{\partial x^{4}}\right) v d x d t \\
& -\int_{t_{a}}^{t_{b}} \int_{c}^{l}\left(\rho A \frac{\partial^{2} u}{\partial t^{2}}+\mathrm{EI} \frac{\partial^{4} u}{\partial x^{4}}\right) v d x d t .
\end{aligned}
$$

Setting (2.15) to zero since $v$ is an arbitrary smooth function satisfying conditions (2.14), the fundamental lemma of the calculus of variations can be applied, and we obtain that $u$ must satisfy the following differential equations:

$$
\begin{array}{ll}
\mathrm{EI} \frac{\partial^{4} u(x, t)}{\partial x^{4}}+\rho A \frac{\partial^{2} u(x, t)}{\partial t^{2}}=0 & \forall t, \forall x \in(0, c), \\
\mathrm{EI} \frac{\partial^{4} u(x, t)}{\partial x^{4}}+\rho A \frac{\partial^{2} u(x, t)}{\partial t^{2}}=0 & \forall t, \forall x \in(c, l) .
\end{array}
$$

Next we remove the restrictions (2.14); and since $u$ must satisfy (2.16) and (2.17), (2.13) reduces to

$$
\begin{aligned}
\delta F(u ; v)= & \int_{t_{a}}^{t_{b}}\left(-r_{1} \frac{\partial u(0, t)}{\partial x}+\mathrm{EI} \frac{\partial^{2} u(0, t)}{\partial x^{2}}\right) \frac{\partial v(0, t)}{\partial x} d t \\
& +\int_{t_{a}}^{t_{b}}\left(-t_{1} u(0, t)-\mathrm{EI} \frac{\partial^{3} u(0, t)}{\partial x^{3}}\right) v(0, t) d t \\
& +\int_{t_{a}}^{t_{b}}\left(-r_{c} \frac{\partial u(c, t)}{\partial x}-\mathrm{EI} \frac{\partial^{2} u\left(c^{-}, t\right)}{\partial x^{2}}+\mathrm{EI} \frac{\partial^{2} u\left(c^{+}, t\right)}{\partial x^{2}}\right) \frac{\partial v(c, t)}{\partial x} d t \\
& +\int_{t_{a}}^{t_{b}}\left(-t_{c} u(c, t)+\mathrm{EI} \frac{\partial^{3} u\left(c^{-}, t\right)}{\partial x^{3}}-\mathrm{EI} \frac{\partial^{3} u\left(c^{+}, t\right)}{\partial x^{3}}\right) v(c, t) d t \\
& +\int_{t_{a}}^{t_{b}}\left(-r_{2} \frac{\partial u(l, t)}{\partial x}-\mathrm{EI} \frac{\partial^{2} u(l, t)}{\partial x^{2}}\right) \frac{\partial v(l, t)}{\partial x} d t \\
& +\int_{t_{a}}^{t_{b}}\left(-t_{2} u(l, t)+\mathrm{EI} \frac{\partial^{3} u(l, t)}{\left.\partial x^{3}\right) v(l, t) d t}\right.
\end{aligned}
$$

The expression (2.18) must vanish for the function $u$ corresponding to the actual motion of the mechanical system under study, and as the functions $v(0, t), \partial v(0, t) / \partial x, v(l, t), \partial v(l, t) / \partial x, v(c, t), \partial v(c, t) / \partial x$, and the interval $\left[t_{a}, t_{b}\right]$ are arbitrary, equating (2.18) to zero leads to the natural 
510 Eigenfrequencies of generally restrained beams

boundary conditions of the problem:

$$
\begin{aligned}
r_{1} \frac{\partial u(0, t)}{\partial x} & =\mathrm{EI} \frac{\partial^{2} u(0, t)}{\partial x^{2}} \\
t_{1} u(0, t) & =-\mathrm{EI} \frac{\partial^{3} u(0, t)}{\partial x^{3}} \\
r_{c} \frac{\partial u(c, t)}{\partial x} & =\mathrm{EI}\left(-\frac{\partial^{2} u\left(c^{-}, t\right)}{\partial x^{2}}+\frac{\partial^{2} u\left(c^{+}, t\right)}{\partial x^{2}}\right) \\
t_{c} u(c, t) & =\mathrm{EI}\left(\frac{\partial^{3} u\left(c^{-}, t\right)}{\partial x^{3}}-\frac{\partial^{3} u\left(c^{+}, t\right)}{\partial x^{3}}\right) \\
r_{2} \frac{\partial u(l, t)}{\partial x} & =-\mathrm{EI} \frac{\partial^{2} u(l, t)}{\partial x^{2}}, \quad t_{2} u(l, t)=\mathrm{EI} \frac{\partial^{3} u(l, t)}{\partial x^{3}}
\end{aligned}
$$

\section{Determination of the exact solution}

Using the well-known method of separation of variables, one assumes as a solution of (2.16) the expression of the form

$$
u^{-}(x, t)=\sum_{n=1}^{\infty} u_{n}^{-}(x) T(t)
$$

Similarly, for (2.17), we write

$$
u^{+}(x, t)=\sum_{n=1}^{\infty} u_{n}^{+}(x) T(t) .
$$

The functions $u_{n}^{-}(x)$ and $u_{n}^{+}(x)$ denote the corresponding $n$th mode of natural vibration and are, respectively, given by

$$
\begin{aligned}
& u_{n}^{-}(x)=A_{1} \cosh k x+A_{2} \sinh k x+A_{3} \cos k x+A_{4} \sin k x, \\
& u_{n}^{+}(x)=A_{5} \cosh k x+A_{6} \sinh k x+A_{7} \cos k x+A_{8} \sin k x,
\end{aligned}
$$

where the parameter $k$ is given by $k=\left(\sqrt{\rho A / \mathrm{EI}} \omega_{n}\right)^{1 / 2}$.

Substituting (3.3) in (3.1) and (3.2) and then in the boundary conditions (2.19) and in the continuity conditions (2.5), one obtains a set of eight homogeneous equations in the constants $A_{i}$. Since the system is homogeneous for the existence of a nontrivial solution, the determinant of coefficients must be equal to zero. This procedure yields the frequency equation

$$
G\left(R_{i}, T_{i}, R_{c}, T_{c}, \lambda, c\right)=0,
$$


where

$$
\begin{gathered}
R_{i}=\frac{r_{i} l}{(\mathrm{EI})}, \quad T_{i}=\frac{t_{i} l^{3}}{(\mathrm{EI})}, \quad i=1,2, \\
R_{c}=\frac{r_{c} l}{(\mathrm{EI})}, \quad T_{c}=\frac{t_{c} l^{3}}{(\mathrm{EI})}, \\
\lambda=k l, \quad c=\left(\sqrt{\frac{\rho A}{\mathrm{EI}}} \omega l\right)^{1 / 2} .
\end{gathered}
$$

\section{Analysis of particular cases}

Since the analytical expression of (3.4) is extremely complex, it is not included, but since it can be used to obtain special cases by substituting limiting values of the restraint parameters $R_{i}, T_{i}, i=1,2, R_{c}$, and $T_{c}$, some particular analytical expressions will be included.

(i) Boundary conditions: RR-F (one end rotationally restrained and the other free, $\left.T_{1} \rightarrow \infty, R_{2} \rightarrow 0, T_{2} \rightarrow 0, R_{c} \rightarrow 0, T_{c} \rightarrow 0\right)$.

Frequency equation:

$$
\lambda(\sinh \lambda \cos \lambda-\sin \lambda \cosh \lambda)+R_{1}(1+\cos \lambda \cosh \lambda)=0 .
$$

(ii) Boundary conditions: TR-TR (both ends translationally restrained $\left.R_{1} \rightarrow 0, R_{2} \rightarrow 0, R_{c} \rightarrow 0, T_{c} \rightarrow 0\right)$.

Frequency equation:

$$
\begin{aligned}
& \lambda^{6}(1-\cos \lambda \cosh \lambda)+T_{2} \lambda^{3}(-\sin \lambda \cosh \lambda+\sinh \lambda \cos \lambda) \\
& \quad+T_{1} \lambda^{3}(\sinh \lambda \cos \lambda-\sin \lambda \cosh \lambda)+2 T_{1} T_{2} \sinh \lambda \sin \lambda=0 .
\end{aligned}
$$

(iii) Boundary conditions: SLIDING-ER (one end sliding and the other elastically restrained against rotation and translation, $R_{1} \rightarrow \infty, T_{1} \rightarrow 0$, $\left.R_{c} \rightarrow 0, T_{c} \rightarrow 0\right)$.

Frequency equation:

$$
\begin{aligned}
& 2\left(\lambda T_{2} \cos \lambda \cosh \lambda-R_{2} \lambda^{3} \sinh \lambda \sin \lambda\right) \\
& \quad+(\sinh \lambda \cos \lambda+\cosh \lambda \sin \lambda)\left(R_{2} T_{2}-\lambda^{4}\right)=0 .
\end{aligned}
$$

\section{Numerical results}

The first five natural frequencies of free vibration of beams with several complicating effects were obtained by using the following strategy. When the values of the parameters $R_{i}, T_{i}, R_{c}, T_{c}$, and $c$ are given, (3.4) reduces to $\bar{G}(\lambda)=0$. A first approximation of the roots of this equation was 
512 Eigenfrequencies of generally restrained beams

TABLE 5.1. Values of the coefficient $\lambda_{1}$ of a cantilever beam with an intermediate point elastically restrained against rotation and translation, $T_{1}=\infty, R_{1}=\infty, T_{2}=0, R_{2}=0$, and $c=0.6$.

\begin{tabular}{rr|cccccc}
\hline & \multicolumn{5}{|c}{$T_{c}$} \\
& & 0 & 1 & 10 & 100 & 1000 & 10000 \\
\hline 0 & 1.87510407 & 1.90645722 & 2.13028597 & 2.93657102 & 3.57232073 & 3.67174004 \\
& 1 & 2.06654909 & 2.09118908 & 2.27624824 & 3.02368609 & 3.65356416 & 3.75239457 \\
$R_{c}$ & 10 & 2.60875718 & 2.62383401 & 2.74617885 & 3.37789697 & 4.03322217 & 4.13616565 \\
& 100 & 2.94991835 & 2.96250788 & 3.06733852 & 3.67937709 & 4.44669639 & 4.56946848 \\
1000 & 3.00457788 & 3.01689906 & 3.11984386 & 3.73240031 & 4.53274975 & 4.66229891 \\
10000 & 3.01037153 & 3.02266576 & 3.12542146 & 3.73807988 & 4.54227057 & 4.67263743 \\
\hline
\end{tabular}

TABLE 5.2. Values of coefficients $\lambda_{1}$ for a beam with both ends and the intermediate point elastically restrained against rotation and translation.

\begin{tabular}{rr|ccccc}
\hline & & \multicolumn{5}{|c}{$T_{c}$} \\
& & 0 & 1 & 10 & 100 & 1000 \\
\hline & 0 & 1.72043695 & 1.76837312 & 2.08199639 & 3.08486980 & 3.20873280 \\
& 1 & 1.73326078 & 1.78041921 & 2.09082061 & 3.12149362 & 3.33630498 \\
$R_{c}$ & 10 & 1.76617200 & 1.81126557 & 2.11253599 & 3.15326549 & 3.89970682 \\
& 100 & 1.78185396 & 1.82592282 & 2.12240538 & 3.15839242 & 4.34988176 \\
& 1000 & 1.78402861 & 1.82795307 & 2.12374977 & 3.15892488 & 4.35002422 \\
\hline
\end{tabular}

determined by means of a graphical procedure. The corresponding numerical values with an accuracy of 15 digits were obtained by applying the classical bisection method and then rounded to eight decimal digits.

Some of these were compared with those available in the literature. The results are presented in a tabular form in Tables 5.1, 5.2, 5.3, 5.4, 5.5, and 5.6.

\section{Translationally and rotationally constrained cantilever beam}

Table 5.1 depicts the values of the coefficient $\lambda_{1}$ of a cantilever beam with an intermediate point elastically restrained against rotation and translation. The values obtained with the present approach, when rounded to five decimal digits, show a complete agreement with the values reported by Lau [13]. 
TABLE 5.3. Values of coefficients $\lambda_{2}$ for a beam with both ends and the intermediate point elastically restrained against rotation and translation.

\begin{tabular}{rr|ccccc}
\hline & & \multicolumn{5}{|c}{$T_{c}$} \\
& & 0 & 1 & 10 & 100 & 1000 \\
\hline & 0 & 3.22334788 & 3.22346332 & 3.22460001 & 3.27769943 & 4.35024819 \\
& 1 & 3.34730310 & 3.34737250 & 3.34804509 & 3.36944527 & 4.35025349 \\
$R_{c}$ & 10 & 3.90227826 & 3.90228315 & 3.90232854 & 3.90296435 & 4.35031637 \\
& 100 & 4.51301613 & 4.51301773 & 4.51303213 & 4.51318339 & 4.51602489 \\
& 1000 & 4.64793800 & 4.64794190 & 4.64797701 & 4.64833088 & 4.65211572 \\
\hline
\end{tabular}

TABLE 5.4. Values of coefficients $\lambda_{3}$ for a beam with both ends and the intermediate point elastically restrained against rotation and translation.

\begin{tabular}{rr|ccccc}
\hline & & \multicolumn{5}{|c}{$T_{c}$} \\
& & 0 & 1 & 10 & 100 & 1000 \\
\hline & 0 & 6.06090936 & 6.06297669 & 6.08160554 & 6.26861751 & 7.70206094 \\
& 1 & 6.06131847 & 6.06338589 & 6.08201554 & 6.26903564 & 7.70295117 \\
$R_{c}$ & 10 & 6.06395142 & 6.06601882 & 6.08464827 & 6.27166813 & 7.70772262 \\
& 100 & 6.06943070 & 6.07149472 & 6.09009401 & 6.27684185 & 7.71423506 \\
& 1000 & 6.07129751 & 6.07335937 & 6.09193939 & 6.27851772 & 7.71581204 \\
\hline
\end{tabular}

TABLE 5.5. Values of coefficients $\lambda_{4}$ for a beam with both ends and the intermediate point elastically restrained against rotation and translation.

\begin{tabular}{rr|ccccc}
\hline & & \multicolumn{4}{c}{$T_{c}$} \\
& & 0 & 1 & 10 & 100 & 1000 \\
\hline & 0 & 9.08972148 & 9.08973195 & 9.08982653 & 9.09080408 & 9.10521528 \\
& 1 & 9.14024985 & 9.14026019 & 9.14035354 & 9.14131701 & 9.15524739 \\
$R_{c}$ & 10 & 9.48041713 & 9.48042702 & 9.48051627 & 9.48142974 & 9.49324713 \\
& 100 & 10.25960952 & 10.25962165 & 10.25973098 & 10.26083269 & 10.27273276 \\
1000 & 10.53799639 & 10.53801108 & 10.53814340 & 10.53946999 & 10.55305237 \\
\hline
\end{tabular}

Translationally and rotationally constrained beam at both ends and at an intermediate point

Tables 5.2, 5.3, 5.4, 5.5, and 5.6 depict the values of coefficients $\lambda_{i}, i=$ $1, \ldots, 5$, for a general restrained beam. Both ends and the intermediate 
514 Eigenfrequencies of generally restrained beams

TABLE 5.6. Values of coefficients $\lambda_{5}$ for a beam with both ends and the intermediate point elastically restrained against rotation and translation.

\begin{tabular}{rr|ccccc}
\hline & \multicolumn{5}{|c}{$T_{c}$} \\
& & 0 & 1 & 10 & 100 & 1000 \\
\hline & 0 & 12.15273465 & 12.15300155 & 12.15540541 & 12.17961523 & 12.43664656 \\
& 1 & 12.15362210 & 12.15388894 & 12.15629225 & 12.18049668 & 12.43748474 \\
$R_{c}$ & 10 & 12.16024052 & 12.16050678 & 12.16290491 & 12.18705823 & 12.44361796 \\
& 100 & 12.18208455 & 12.18234743 & 12.18471518 & 12.20856803 & 12.46251085 \\
1000 & 12.19397508 & 12.19423521 & 12.19657823 & 12.22018715 & 12.47203118 \\
\hline
\end{tabular}

point are elastically restrained against rotation and translation $\left(T_{1}=1\right.$, $R_{1}=100, T_{2}=10, R_{2}=10$, and $c=1 / 2$ ).

\section{Conclusions}

Exact frequency expressions for generally restrained beams with intermediate elastic constraints were derived. Numerical results for the first five natural frequencies have been presented in tabular form.

Several particular cases were solved and the results obtained were compared with previously published results to demonstrate the accuracy and flexibility of the present approach. Excellent agreement was obtained between the present results and the comparison exact values.

It can also be seen, from the results presented, that both the rotational and the translational restraints at the intermediate point have a significant effect on the frequencies and that the translational restraint generally has greater influence on these frequencies than the rotational restraint.

The procedure presented has a great flexibility and excellent accuracy and constitutes an efficient tool for the rapid and inexpensive determination of natural frequencies in an important number of beam vibrating problems being, in consequence, of interest in design works.

Boundary conditions containing the function $u$ and derivatives of $u$ of orders not greater than $m-1$ are called stable or geometric for a differential equation of order $2 m$, and those containing derivatives of orders higher than $m-1$ are called unstable or natural [23]. Thus, if $0 \leq$ $r_{i}, r_{c}<\infty$, and if $0 \leq t_{i}, t_{c}<\infty, i=1,2$, conditions (2.19) are unstable. It is well known that when using the Ritz method, we choose a sequence of functions $v_{i}$ which constitute a base in the space of homogeneous stable boundary conditions (see [23]), so in this case there is no need to subject the functions $v_{i}$ to the natural boundary conditions (2.19). This is a 
very important characteristic of the mentioned variational method in the determination of approximate solutions of the problem under study.

\section{Acknowledgments}

The present study has been sponsored by Consejo de Investigación, Project no. 916 (UNSA). The authors are grateful to the referee for valuable comments and suggestions. Ricardo Oscar Grossi is a research member of CONICET, Argentina.

\section{References}

[1] B. Arenas and R. O. Grossi, Vibration frequencies for a beam with a rotational restraint in an adjustable position, Applied Acoustics 57 (1999), 197-202.

[2] R. Chun, Free vibration of a beam with one end spring-hinged and the other free, Journal of Applied Mechanics 39 (1972), 1154-1155.

[3] H. Cortinez and P. A. A. Laura, Vibration and buckling of a non-uniform beam elastically restrained against rotation at one end and with concentrated mass at the other, J. Sound Vibration 99 (1985), 144-148.

[4] P. Goel, Free vibrations of a beam-mass system with elastically restrained ends, J. Sound Vibration 47 (1976), 9-14.

[5] - Transverse vibrations of tapered beams, J. Sound Vibration 47 (1976), $1-7$.

[6] A. Grant, Vibration frequencies for a uniform beam with one end elastically supported and carrying a mass at the other end, Journal of Applied Mechanics 42 (1975), 878-880.

[7] R. O. Grossi, On the role of natural boundary conditions in the Ritz method, International Journal of Mechanical Engineering Education 16 (1988), 207-210.

[8] R. O. Grossi, A. Aranda, and R. B. Bhat, Vibration of tapered beams with one end spring hinged and the other end with tip mass, J. Sound Vibration 160 (1993), 175-178.

[9] R. O. Grossi and R. B. Bhat, A note on vibrating tapered beams, J. Sound Vibration 147 (1991), 174-178.

[10] R. O. Grossi and P. A. A. Laura, Further results on a vibrating beam with a mass and spring at the end subjected to an axial force, J. Sound Vibration 84 (1982), 593-594.

[11] M. S. Hess, Vibration frequencies for a uniform beam with central mass and elastic supports, Journal of Applied Mechanics 31 (1964), 556-558.

[12] C. Hibbeler, Free vibrations of a beam supported with unsymmetrical springhinges, Journal of Applied Mechanics 42 (1975), 501-502.

[13] J. H. Lau, Vibration frequencies and mode shapes for a constrained cantilever, Journal of Applied Mechanics 57 (1984), 182-187.

[14] P. A. A. Laura, R. O. Grossi, and S. Alvarez, Transverse vibrations of a beam elastically restrained at one end and with a mass and spring at the other subjected to an axial force, Nuclear Engineering and Design 74 (1982), 299-302. 
516 Eigenfrequencies of generally restrained beams

[15] P. A. A. Laura and R. H. Gutierrez, Vibration of an elastically restrained cantilever beam of varying cross section with tip mass of finite length, J. Sound Vibration 108 (1986), 123-131.

[16] W. Lee, Vibration frequencies for a uniform beam with one end spring hinged and carrying a mass at the other free end, Journal of Applied Mechanics 40 (1973), 813-815.

[17] H. Mabie and C. B. Rogers, Transverse vibrations of tapered cantilever beams with end support, J. Acoust. Soc. Amer. 44 (1968), 1739-1741.

[18] _ Transverse vibrations of double-tapered cantilever beams, J. Acoust. Soc. Amer. 57 (1972), 1771-1774.

[19] - Transverse vibrations of double-tapered cantilever beams with end support and with end mass, J. Acoust. Soc. Amer. 55 (1974), 986-991.

[20] R. Maurizi, R. Rossi, and J. Reyes, Vibration frequencies for a uniform beam with one end spring hinged and subjected to a translational restraint at the other end, J. Sound Vibration 48 (1976), 565-568.

[21] L. Nallim and R. O. Grossi, A general algorithm for the study of the dynamical behaviour of beams, Applied Acoustics 57 (1999), 345-356.

[22] C. K. Rao and S. Mirza, Note on vibrations of generally restrained beams, J. Sound Vibration 130 (1989), 453-465.

[23] K. Rektorys, Variational Methods in Mathematics, Science and Engineering, D. Reidel, Dordrecht, 1980.

[24] A. Rutemberg, Vibration frequencies for a uniform cantilever with a rotational constraint at a point, Journal of Applied Mechanics 45 (1978), 422-423.

[25] R. Szilard, The Theory and Analysis of Plates, Prentice-Hall, New Jersey, 1963.

[26] J. L. Troutman, Variational Calculus and Optimal Control, Undergraduate Texts in Mathematics, Springer-Verlag, New York, 1996.

Ricardo Oscar Grossi: ICMASA, Facultad de Ingeniería, Universidad Nacional de Salta, Avenida Bolivia 5150, 4440 Salta, Argentina

E-mail address: grossiro@unsa.edu.ar

Carlos Marcelo Albarracín: ICMASA, Facultad de Ingeniería, Universidad Nacional de Salta, Avenida Bolivia 5150, 4440 Salta, Argentina 


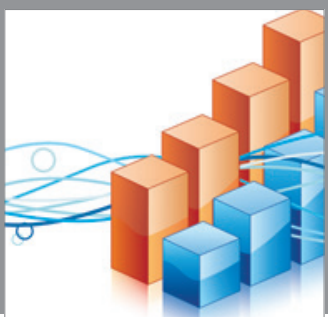

Advances in

Operations Research

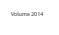

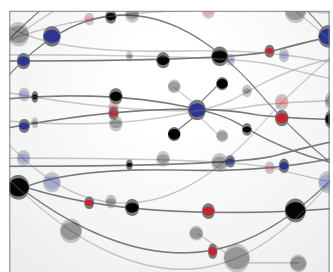

\section{The Scientific} World Journal
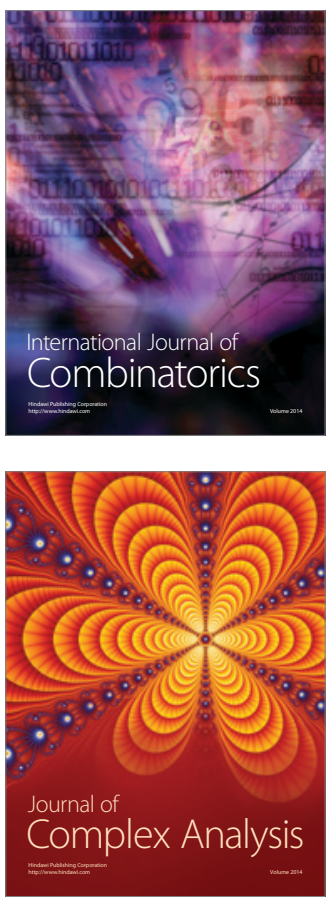

International Journal of

Mathematics and

Mathematical

Sciences
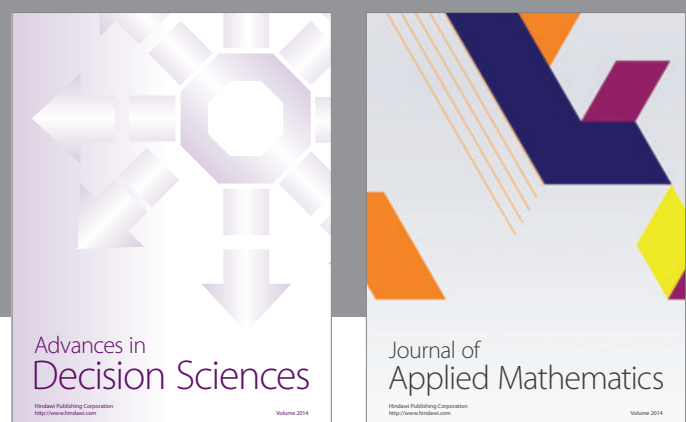

Journal of

Applied Mathematics
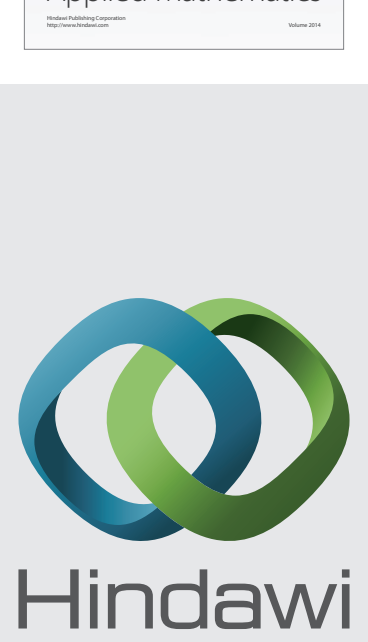

Submit your manuscripts at http://www.hindawi.com
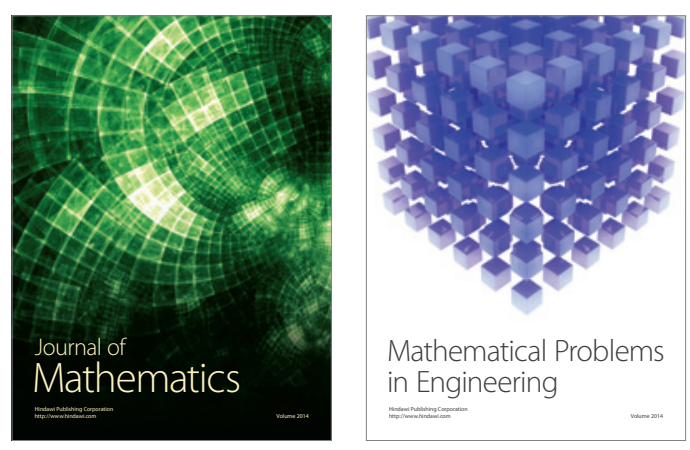

Mathematical Problems in Engineering
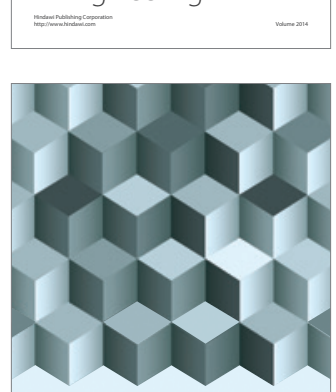

Journal of

Function Spaces
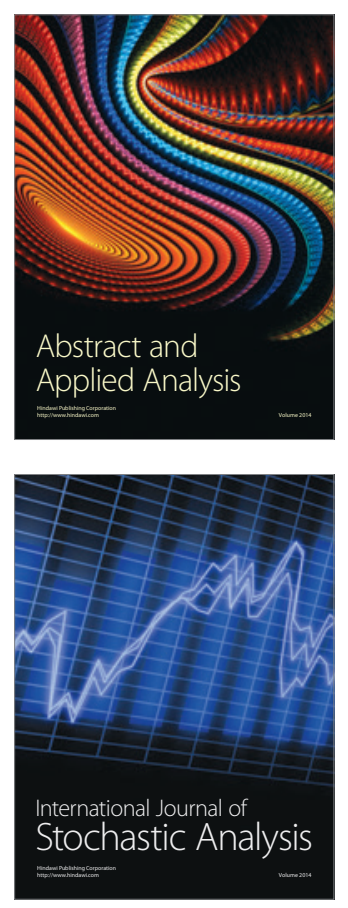

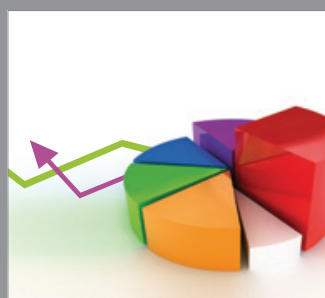

ournal of

Probability and Statistics

Promensencen
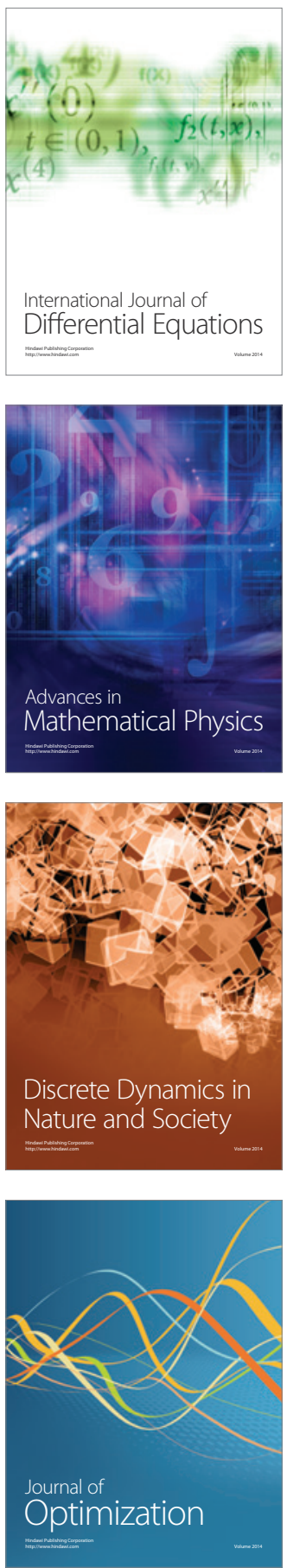